\title{
Experience of a Videoconference System with Medical Information Desktop Sharing for Radiation Therapy and Evaluation of Its Usefulness
}

\author{
Shohei Kawashiro, Yasuhito Hagiwara, Ibuki Ota, Mayumi Ichikawa, \\ Misako Miwa, Yuuki Kuroda, Kenji Nemoto \\ Department of Radiation Oncology, Yamagata University Faculty of Medicine, Yamagata, Japan \\ Email: shohei.kawashiro@gmail.com
}

Received November 8, 2013; revised December 3, 2013; accepted January 2, 2014

Copyright (C) 2014 Shohei Kawashiro et al. This is an open access article distributed under the Creative Commons Attribution License, which permits unrestricted use, distribution, and reproduction in any medium, provided the original work is properly cited. In accordance of the Creative Commons Attribution License all Copyrights (C) 2014 are reserved for SCIRP and the owner of the intellectual property Shohei Kawashiro et al. All Copyright (C) 2014 are guarded by law and by SCIRP as a guardian.

\begin{abstract}
Purpose: To present our videoconference system with medical information desktop sharing for radiation therapy and report initial experience using the system and its usefulness. Materials and Methods: A videoconference system for radiation therapy enables radiation oncologists sent to affiliated hospitals to consult a more experienced board-certified doctor at our institution while sharing the same display showing a patient's information, radiology information system (RIS), and radiation therapy planning (RTP) system. We evaluated cases discussed in videoconferences to determine the influence of the system on treatment policies and radiation therapy plans. Results: From December 2012 to March 2013, treatment policies for 56 cases and radiation therapy plans for 50 cases were discussed in videoconferences. As for treatment policies, no change was made in treatment policy for 33 cases $(59 \%)$, and minor and major changes were made for $16(29 \%)$ cases and $7(12 \%)$ cases, respectively. Radiation therapy plans for 50 cases were checked. No change was needed for 32 cases (64\%), and minor and major changes were needed for $17(34 \%)$ cases and $1(2 \%)$ case, respectively. Conclusion: The videoconference system can be effective for improving the quality of radiation therapy.
\end{abstract}

\section{KEYWORDS}

Radiation Therapy; Shortage of Radiation Oncologists; Videoconference; Desktop Sharing; Telemedicine

\section{Introduction}

The number of patients undergoing radiation therapy in Japan has been increasing recently [1]. A shortage of board-certified radiation oncologists is a major concern, especially in rural areas, and many radiation oncologists perform part-time duties at other institutions as well as at those to which they belong. There are not enough boardcertified radiation oncologists at our institution to send as full-time staff to its affiliated hospitals. Radiation oncologists with less experience sometimes encounter cases for which it is difficult to decide a treatment policy and to make a treatment plan, and treatment plans should be checked by more experienced board-certified radiation oncologists for safety. In order to resolve this problem, we set up a videoconference system at our institution and its affiliated hospitals in June 2012. This system enabled radiation oncologists sent to the affiliated hospitals to consult a more experienced board-certified doctor at our institution as they can share the same display showing a patient's medical record, radiological information system (RIS), and radiation therapy planning (RTP) system. Here, we present our videoconference system for radiation therapy and report our initial experience with the system and its usefulness.

\section{Materials and Methods}

\subsection{System}

The system we introduced at our institution and its affiliated hospitals was the KX-VC Series of an HD Visual 
Communication Unit (Panasonic Corporation, Osaka, Japan). It consists of a main unit, a display, a video camera, a microphone, and a router. This system was set up at our institution and its three affiliated hospitals. These hospitals are all located in the same prefecture. The radiation oncologists at our institution are sent to the three affiliated hospitals as part-time staff once or twice a week, as there is no full-time radiation oncologist at any of the three hospitals. The systems at all hospitals are connected to each other via a virtual private network (VPN). A schematic image of the system is shown in Figure 1. Just like making a phone call, the radiation oncologists sent to the affiliated hospitals can consult a more experienced board-certified doctor at our institution regarding treatment policies and have their treatment plans checked. The displays of the video camera, the electronic medical records, and the RTP system can be switched. The radiation oncologists both at our institution and the affiliated hospital can see the same display, share a patient's information, and conduct a conference at the same time. The monitor in this system can display images in full high-definition resolution, and the data transmission rate is sufficiently high for images such as CT, MRI and PET images to be clearly shown. Scenes of an actual videoconference are shown in Figure 2.

\subsection{Evaluation of the Usefulness of the System}

During the period from December 2012 to March 2013, treatment policies for 56 cases were discussed in videoconferences between our institution and its affiliated hospitals. Among the 56 cases, decisions were made to perform radiation therapy in 50 cases, and plans for radiation therapy were actually made by the radiation oncologists sent to the affiliated hospitals and checked by the more experienced board-certified radiation oncologists at our institution in videoconferences. We evaluated these cases discussed in videoconferences to determine the influence on treatment policies and radiation therapy plans. Ogawa et al. [2] classified changes made in planning into three categories for evaluation of their remote RTP system. Based on this classification, we also categorized the cases discussed in videoconferences according to the magnitude of changes in treatment policies and radiation therapy plans: no change, minor change, or major change.

\section{Results}

The influence of the videoconference on treatment policies was as follows. Of the 56 cases discussed in videoconferences, no change was made in treatment policy for 33 cases (59\%), minor changes were made for 16 cases (29\%), and major changes were made for 7 cases (12\%). Minor changes included slight changes of treatment policy such as changes of dose fraction and field size in treatment policies. And Major changes included the cases for which radiation therapy was not indicated, other treatment such as surgery was recommended, or relatively large changes of field size and/or dose fraction was indicated in treatment policies.

During the period from December 2012 to March 2013, 117 radiation therapy plans were made at the affiliated hospitals in total. Of these cases, 50 cases were checked in videoconferences. The target sites and changes made in videoconferences are shown in Table 1 . No change was needed for 32 cases (64\%). Minor change was needed for 17 cases (34\%) and major change was needed in only 1 case (2\%). The standards for which to judge whether or not it is necessary to revise treatment plans are as follows: 1) The contours of tumors and organs at risk are correctly delineated. 2) The number of beams and the beam angles are appropriate. 3) The dose distribution and the dose volume histogram are acceptable. The minor changes included small revisions such as adjustments of multilieaf collimators to an extent that the response of treatment did not seem to be influenced. The

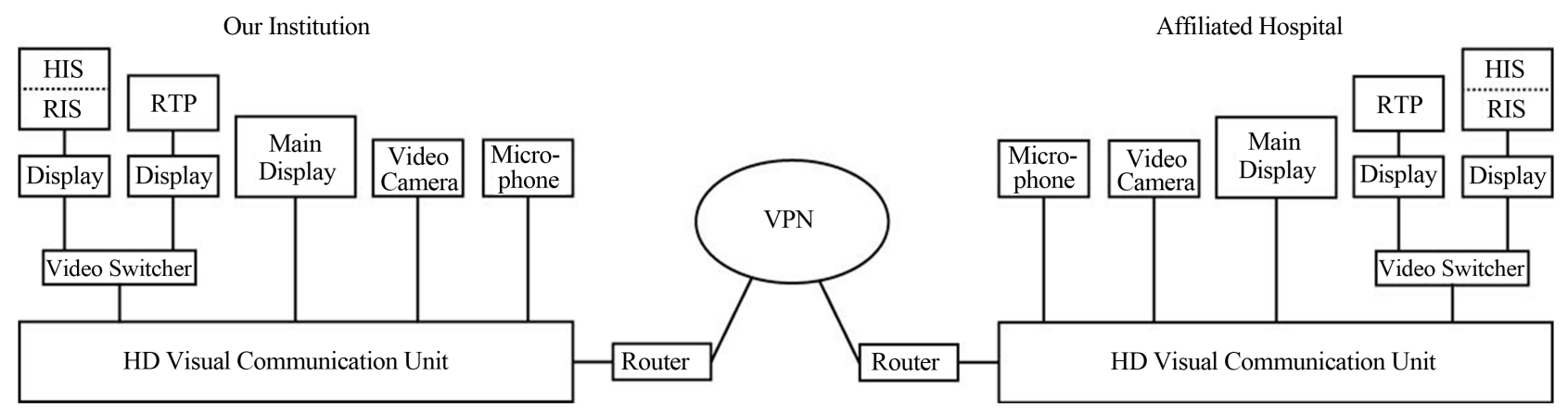

Figure 1. Composition of the videoconference system. HD Visual Communication Units are connected via a VPN between our institution and its affiliated hospitals. The displays of HIS/RIS and the RTP system are connected to the Units. The images of HIS/RIS, RTP system, and video camera can be switched and the same desktop can be shared on the main display between our institution and the affiliated hospital. VPN = virtual private network, HIS = hospital information system, RIS = radiology information system, $\mathrm{RTP}$ = radiation therapy planning. 
Table 1. Changes made in radiation therapy plans checked in videoconferences.

\begin{tabular}{|c|c|c|c|c|c|c|}
\hline \multirow[b]{2}{*}{ Site } & \multicolumn{4}{|c|}{ Plans checked in videoconferences (A) } & \multirow{2}{*}{$\begin{array}{l}\text { Plans not checked in } \\
\text { videoconferences (B) }\end{array}$} & \multirow{2}{*}{$\begin{array}{l}\text { All cases } \\
(\mathrm{A})+(\mathrm{B})\end{array}$} \\
\hline & C1 & $\mathrm{C} 2$ & C3 & Total & & \\
\hline Esophagus & 8 & 3 & 0 & 11 & 2 & 13 \\
\hline Bone metastasis & 4 & 4 & 0 & 8 & 5 & 13 \\
\hline Breast & 4 & 4 & 0 & 8 & 34 & 42 \\
\hline Head and neck & 4 & 4 & 0 & 8 & 3 & 11 \\
\hline Brain & 2 & 1 & 0 & 3 & 4 & 7 \\
\hline Prostate & 2 & 0 & 0 & 2 & 8 & 10 \\
\hline Mediastinum & 2 & 0 & 0 & 2 & 1 & 3 \\
\hline Liver & 2 & 0 & 0 & 2 & 0 & 2 \\
\hline Lymphoma & 0 & 0 & 1 & 1 & 0 & 1 \\
\hline Lung & 0 & 1 & 0 & 1 & 3 & 4 \\
\hline Lymph node metastasis & 1 & 0 & 0 & 1 & 4 & 5 \\
\hline Pelvis & 1 & 0 & 0 & 1 & 3 & 4 \\
\hline Spinal cord & 1 & 0 & 0 & 1 & 0 & 1 \\
\hline Pancreas & 1 & 0 & 0 & 1 & 0 & 1 \\
\hline Total & $32(64 \%)$ & $17(34 \%)$ & $1(2 \%)$ & $50(100 \%)$ & 67 & 117 \\
\hline
\end{tabular}

C1 = no changes made in plan, C2 = minor changes made in plan, C3 = major changes made in plan.

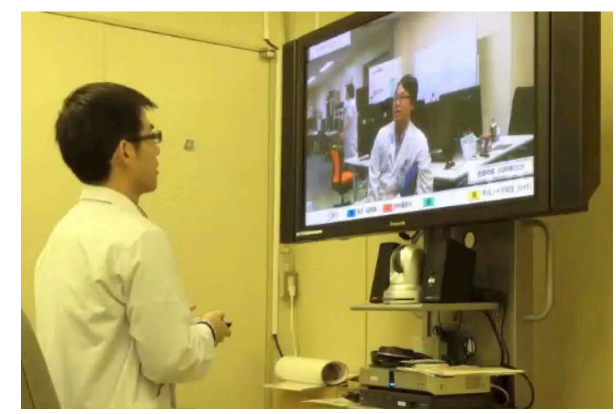

(a)

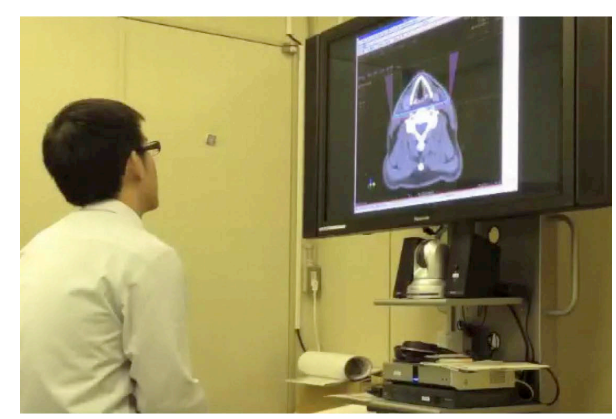

(b)

Figure 2. Scenes of a videoconference. (a) The radiation oncologist sent to an affiliated hospital is consulting a more experienced board-certified doctor at our institution regarding a treatment policy; (b) A radiation therapy plan is checked as they are sharing the desktop of the radiation therapy planning system on the main display. one case for which major change was made was a radiation therapy plan for extranodal NK/T-cell lymphoma, nasal type. And it was thought to require a large change in the irradiation field because of the dose distribution to the target and organs at risk, and re-planning thus seemed necessary.

\section{Discussion}

\subsection{Telemedicine for Radiation Therapy}

With advances in information and communication technology (ICT), the potential for applications of ICT in the field of radiation oncology also seems to be expanding. Our system reported here is one example of utilization of ICT for radiation therapy. Use of ICT for telemedicine in radiation therapy has been reported in the literature. Olsen et al. [3] described a telemedicine system for radiation therapy. They classified telemedicine requirements and applications into three levels. This classification of levels is presented in Table 2. Our videoconference system may belong to level 1 of the classification of Olsen et al., though the classification was proposed before electronic medical records and RIS became available, and it is not known whether desktop sharing was assumed by the classification. Our main needs for telemedicine in radiation therapy are 1) consultation regarding treatment policies for radiation therapy with board-certified 
Table 2. Telemedicine requirements according to the classification of Olsen et al. [3]

\begin{tabular}{rcccc}
\hline & Video conference & Image display (wide screen) & Database replication, on request & “Real-time” remote operations \\
\hline Level 1 & + & + & - & - \\
Level 2 & + & + & + & - \\
Level 3 & + & + & + & + \\
\hline
\end{tabular}

radiation oncologists in our institution and 2) discussion of treatment plans made by radiation oncologists sent from our institution to the affiliated hospitals. The system reported here has sufficiently realized our needs, though a level 1 system was the simplest in the classification of Olsen et al. It should be emphasized that medical information desktop sharing reported here seems to be the first in the field of radiation oncology.

There are some reports on applications of telemedicine for radiation therapy [4-7]. Suzuki et al. [4] introduced a web-based remote radiation treatment planning system that allowed staff at an affiliated hospital to obtain support from a fully staffed central institution. In their system, they remotely operated the RTP computer using a remote desktop function. Our video conference system was not equipped with a function to make radiation therapy plans remotely; however, we did not need such a function because radiation therapy plans made by a doctor sent to an affiliated hospital could be checked in the videoconference and corrected on the spot. Maguire et al. [5] reported their initial experience with a telemedicine workstation as a method for interactive intensity-modulated radiation therapy (IMRT) planning for patients with advanced head and neck squamous cell carcinoma between a university hospital and a distant regional community hospital. As an example of telemedicine applied to radiation oncology in a developing country, Agrawal et al. [6] reported the establishment of a telemedicine linkage with a remote medical institute to facilitate teaching, training, and carry out collaborative clinical and translational research. Their utilization of telemedicine in radiation oncology also included a videoconference, although the main purpose was resident education.

The utilization of a videoconference system with desktop sharing in a field other than radiation therapy has also reported. Lee et al. [8] reported implementation of a videoconference system between multiple family medicine departments. Their situation was similar to ours in that they had difficulties holding regular conferences at each hospital due to inadequate staff members and residents. For the purpose of solving this problem, they implemented a videoconference system to hold joint conferences between multiple family medicine departments. Although their system resembled our videoconference system in terms of sharing the desktop as we did, their conferences included clinical reviews, journal reviews and staff lectures. Our video conference system reported here is unique in that we discussed treatment policies and radiation therapy plans instead of reviewing past cases. It should be emphasized again that our system with medical information desktop sharing seems to be the first such system in the field of radiation oncology.

\subsection{Information Security and Cost}

In our system, radiation oncologists both at our institution and the affiliated hospitals can simultaneously see the same desktop to share a patient's medical record, radiological images, pathological reports, and so on. The medical records of patients at the affiliated hospitals are accessible only by doctors at the affiliated hospitals. This means the data for the patients themselves are not exchanged between hospitals. In other words, the risk of illegal access to a patient's information is considered to be low. In addition, the initial cost for introducing this videoconference system was not high, about 1 million yen for each institution. Therefore, the videoconference system presented here seems to be feasible in terms of information security and cost.

\subsection{Influence of the Videoconference}

Among the 56 cases for which treatment policies were discussed in videoconferences, 23 cases (40\%) of them were influenced by the discussions overall. Some cases were influenced to such an extent that treatment policies other than radiation therapy were proposed. These discussions probably led to better treatment for patients. As for radiation therapy plans, 50 of the 56 cases discussed in videoconferences were checked by more experienced board-certified radiation oncologists at our institution, and $18(36 \%)$ of those cases required changes. It seems beneficial for a radiation oncologist to experience as many cases as possible to become an expert. In order to realize this at our institution, experience of cases at affiliated hospitals as well as at our institution is required. The videoconference system presented here can contribute both to better treatment for patients and to education for doctors who are training to be experienced radiation oncologists, although the decisions of board-certified radiation oncologists are not necessarily the best. A limitation of this report is that the outcomes of patients are 
not compared between before and after the introduction of the videoconference system. We think that it is difficult to compare the treatment outcomes of patients at this moment because of short follow-up time after the introduction of the videoconference system. We would like to analyze the outcomes in the near future.

\subsection{Evaluation by the Doctors}

Some advantages and issues to be improved were pointed out by the radiation oncologists who actually used this system at our institution and its affiliated hospitals. First, the advantages are as follows: 1) Treatment policies can be made and/or corrected immediately, as the information of medical records, radiological images, results of pathological examinations, etc. are simultaneously shared by the radiation oncologists both at our institution and the affiliated hospital. 2) Radiation therapy plans can be checked and corrected on the spot by switching the display to the RTP system. This can reduce the time needed to start radiation therapy for the patient. 3) Radiation therapy at the affiliated hospitals can be provided safely due to the videoconference. On the other hand, issues to be improved are as follows: 1 ) It is difficult for a radiation oncologist requesting a consultation to know beforehand whether a more experienced board-certified radiation oncologist can cope with the case at that time. 2) The board-certified radiation oncologist must temporarily stop his/her duties if there is a sudden request for consultation. 3) When a radiation therapy plan is checked, it is sometimes difficult to orally convey details of corrections because the RTP system can be operated only by the radiation oncologist who asks for a consultation. Although there are some points to be improved, radiation oncologists who used this videoconference system for radiation therapy seemed to be generally satisfied with the system.

\section{Conclusion}

The videoconference system with medical information desktop sharing presented here seemed to contribute to better treatment for patients undergoing radiation therapy and to education for radiation oncologists. The system can be effective for improving the quality of radiation therapy.

\section{REFERENCES}

[1] H. Numasaki, M. Nishio, H. Ikeda, K. Sekiguchi, N. Kamikonya, M. Koizumi, et al., “Japanese Structure Survey of Radiation Oncology in 2009 with Special Reference to Designated Cancer Care Hospitals,” International Journal of Clinical Oncology, Vol. 18, No. 5, 2013, pp. 775-783. http://dx.doi.org/10.1007/s10147-012-0468-7

[2] Y. Ogawa, K. Nemoto, Y. Kakuto, H. Seiji, K. Sakaki, C. Takahashi, et al., "Construction of a Remote Radiotherapy Planning System,” International Journal of Clinical Oncology, Vol. 10, No. 1, 2005, pp. 26-29. http://dx.doi.org/10.1007/s10147-004-0446-9

[3] D. R. Olsen, S. Bruland and B. J. Davis, "Telemedicine in Radiotherapy Treatment Planning: Requirements and Applications," Radiotherapy and Oncology, Vol. 54, No. 3, 2000, pp. 255-259. http://dx.doi.org/10.1016/S0167-8140(99)00185-1

[4] K. Suzuki, Y. Hirasawa, Y. Yaegashi, H. Miyamoto and H. Shirato, “A Web-Based Remote Radiation Treatment Planning System Using the Remote Desktop Function of a Computer Operating System: A Preliminary Report,” Journal of Telemedicine and Telecare, Vol. 15, No. 8, 2009, pp. 414-418. http://dx.doi.org/10.1258/jtt.2009.090409

[5] P. D. Maguire, G. Honaker, C Neal, M. Meyerson, D. Morris, J. Rosenman, et al., "A Bridge between Academic and Community Radiation Oncology Treatment Planning,” Journal of Oncology Practice, Vol. 3, No. 5, 2007, pp. 238-241. http://dx.doi.org/10.1200/JOP.0752001

[6] S. Agrawal, A. K. Maurya, K. Shrivastava, S. Kumar, M. C. Pant and S. K. Mishra, "Training the Trainees in Radiation Oncology with Telemedicine as a Tool in a Developing Country: A Two-Year Audit,” International Journal of Telemedicine and Applications, Vol. 2011, 2011, Article ID: 230670.

http://dx.doi.org/10.1155/2011/230670

[7] T. N. Teslow, R. A. Gilbert, W. H. Grant III, S. Y. Woo, E. B. Butler and J. H. Liem, “A Teleradiology Case Conference System,” Journal of Telemedicine and Telecare, Vol. 1, No. 2, 1995, pp. 95-99.

[8] K. H. Lee, J. Y. Kim, K. Lee, B. Cho, J. H. Yang, E. Goh, et al., "Implementation of a Videoconferencing System between Family Medicine Departments," Korean Journal of Family Medicine, Vol. 32, No. 5, 2011, pp. 311-316. 